\title{
The Stem Cell Quiescence and Niche Signaling is Disturbed in the Hair Follicle of the Hairpoor Mouse, an MUHH Model Mouse.
}

Keonwoo Choi

The Catholic University of Korea

Sang-Hee Park

The Catholic University of Korea

Seo-Yeon Park

The Catholic University of Korea

Sungjoo Kim Yoon ( $\nabla$ sjkyoon@catholic.ac.kr)

The Catholic University of Korea https://orcid.org/0000-0003-2497-729X

\section{Research Article}

Keywords: hairpoor mouse, MUHH, Hair follicle stem cell, Wnt signaling, Alopecia

Posted Date: October 25th, 2021

DOI: https://doi.org/10.21203/rs.3.rs-998550/v1

License: (c) (i) This work is licensed under a Creative Commons Attribution 4.0 International License. Read Full License 


\section{Abstract \\ Background}

Hair follicle stem cells (HFSC) play an essential role in the maintenance of hair homeostasis during the hair cycle in which HFSC remain quiescent most of its duration. The hairpoor mouse $\left(+/ H r^{H p}\right)$, an animal model of Marie-Unna Hypotrichosis (MUHH), exhibits over-expression of Hairless in the bulge, inner root sheath and outer root sheath of HF and exhibits the same phenotype as in MUHH patients manifesting sparse hair with progression to alopecia with age. In this study, we aimed to understand the hair cycle and the status of HFSC during the hair cycle of the hairpoor mouse to delineate the pathogenesis of MUHH.

\section{Methods}

To define the state of the hair follicle, H\&E staining was performed. FACS analysis and immunostaining was utilized at the 1 st and 2nd telogen stages to observe the HFSCs. Label retaining assay was carried out to determine the quiescent state of hair follicle. Expression of factors in involved in the niche signaling and the Wnt signaling was determined using qRT-PCR.

\section{Results}

We found that the number of hair follicle was drastically decreased after 1 st telogen, then followed by the intensified disturbance in the hair cycle with shorter anagen as well as 2 nd telogen in the hairpoor mouse. The number of CD34 expressing bulges as well as cells were dramatically reduced at the telogen of the HFs and the high proliferation of bulge cells was prominent, suggesting the loss of HFSC quiescence in the hairpoor mouse. The increased cell proliferation in HF was reiterated following the synchronization of hair cycle, leading to accelerating HF cycling. The expression of Fgf18 and Bmp6, the factors involved in the HFSC quiescence, was reduced in the HFSC niche of the hairpoor mouse. Furthermore, expression of Wnt signaling molecules including Wnt7b, Wnt10b and Sfrp 1 were disturbed inducing the telogen to anagen transition of HFs in the hairpoor mouse.

\section{Conclusions}

These results indicate that the quiescent state of HFSC is not properly maintained in the hairpoor mouse and consequently leading HFs to the completely disarrayed hair cycle. These findings may provide an understanding of an underlying mechanism by which alopecia develops with age in MUHH patients.

\section{Background}


The hair follicle stem cells (HFSC) are located in the bulge of the hair follicle (HF) and play an important role in the development of hair and maintenance of HF cycle [1, 2]. HF continuously undergoes a cycle of growth (anagen), degeneration (catagen), and rest phases (telogen) during the life time [3]. HF cycling is regulated by a precise interplay of several specific signaling activities [4].

Wnt signaling is a central regulator of hair morphogenesis and hair growth [5]. During the anagen phase, activation of Wnt signaling induces HF cycle via the proliferation and differentiation of HFSC [6] and $W n t 7 b$ plays a role as a critical regulator of HF cycle [7]. Expression of $\beta$-catenin, an intracellular signal transducer of the Wnt signaling pathway, increases at the telogen-anagen transition and stays at the same level throughout the anagen phase [8,9]. While the Wnt signaling induces the activation of HFSC proliferation, bone morphogenetic protein (BMP) signaling plays an important role in maintaining quiescence of HFSC $[10,11]$. During the telogen phase, BMP is highly expressed in the HFSC niche. In the Keratin 6 (K6) positive cells in the inner layer of bulge, bone morphogenetic protein 6 (Bmp6) and fibroblast growth factor 18 (Fgf18) are highly expressed, which are shown to be essential in maintaining quiescence of HFSC [12]. Fgf18 conditional knockout mice display the shortened telogen phase [13] and similarly, overexpression of Noggin, a BMP antagonist, in mouse skin results in a shortened telogen phase and activation of HF growth [14]. Thus, HFSC homeostasis and cyclic activation are controlled by the balance between Wnt, BMP and FGF18 signaling.

Hairless $(H r)$ is a transcriptional co-repressor which down regulates target genes in conjunction with the thyroid hormone receptor, retinoic acid receptor, and vitamin D receptor [15-17]. The Hrgene is expressed in the outer root sheath of HF, and involved in the development of HF by regulating Wnt signaling $[18,19]$. Mutations in the $\mathrm{Hrgene}$ have been reported to be associated with hair loss disorders in humans and mice $[20,21]$. Among them, mutations in the 5'UTR of $H R$ are known to cause Marie-Unna Hypotrichosis (MUHH), a genetic disorder of alopecia [22]. Recently, we reported the mutant mouse called "hairpoor" $\left(\mathrm{Hr}^{\mathrm{H} P}\right)$ with T-to-A transversion substitution at position 403 in the second UORF of 5 'UTR of the $\mathrm{Hr}$ gene (NM_021877) [23]. This mouse displays overexpression of HR and the characteristics of the human $\mathrm{MUHH}$, in which manifests sparse hair in early age and gradual hair loss with age [24]. The hairpoor heterozygotes $\left(+/ H r^{H p}\right)$ displayed shortened hair follicles and abnormal differentiation of keratinocyte. Furthermore, Wnt signaling was shown to be unusually complicated in this mouse [24, 25]. Although these studies have described the characteristics of the hairpoor mice, the status of the HFSC in the HR overexpressing hairpoor mouse has not been fully explored.

In the present study, we examined the hair cycle of the hairpoor mouse and investigated the status of the HFSC. Our findings clearly showed that the quiescence of HFSC was not maintained in the hairpoor mouse, exhibiting the shorter telogen phase and premature induction of anagen phase with proliferation of HFSC, consequently leading to completely disarrayed HF cycle. These phenomena concurred with the down regulation of Fgf18, Bmp6, and Sfrp 1 and up regulation of Wnt7b and Wnt10b expression, presumably leading to failure of appropriate signaling activity in the HFSC niche.

\section{Methods}


Animals

Housing conditions of animals were as previously reported [21]. All procedures conducted in accordance with the Laboratory Animals Welfare Act, the Guide for the Care and Use of Laboratory Animals and the Guidelines and Policies for Rodent Experiments provided by the IACUC (Institutional Animal Care and Use Committee) in the School of Medicine, The Catholic University of Korea. Depilation was achieved by manual plucking of dorsal skin followed by wax strip depilation at $2^{\text {nd }}$ telogen [3].

\section{Histology, immunofluorescence, and Immunocytochemistry}

Skin samples were taken at the $1^{\text {st }}$ telogen (wild type : P21, hairpoor: P19-20), $2^{\text {nd }}$ telogen (wild type : P49, hairpoor: P35), or indicated date. Tissues were fixed in $4 \%$ formaldehyde solution overnight at $4{ }^{\circ} \mathrm{C}$ and embedded in paraffin wax. Paraffin sections $(6 \mu \mathrm{m})$ were prepared and hematoxylin and eosin (H\&E) staining was carried out following a standard protocol [24]. For immunofluorescence or immunohistochemical staining, antigen retrieval was performed in $10 \mathrm{mM}$ sodium citrate (pH 6.0). Skin sections were blocked with $5 \%$ BSA in PBS for $1 \mathrm{hr}$ at room temperature and incubated with primary antibodies overnight at $4{ }^{\circ} \mathrm{C}$. The primary antibodies used were anti-CD34 (1:200, Abcam, MA, USA), antiK15 (1:200, Abcam, MA, USA), anti-NFATc1 (1:100, Santacruz, CA, USA), anti-LEF1 (1:100, Cell Signaling Technology, MA, USA), anti-SOX9 (1:100, Santacruz, CA, USA), anti-TCF4 (1:100, Cell Signaling Technology, MA, USA), anti-Ki67 (1:100, NeoMarkers, CA, USA), anti-BrdU (1:200, Abcam, MA, USA) antiK6(1:100, Abcam, MA, USA), anti-BMP6 (1:100, Cell Signaling Technology, MA, USA), and anti-FGF18 (1:100, Cell Signaling Technology, MA, USA). Alexa Fluor 488 or 594 goat anti-mouse, goat anti-rabbit, goat anti-rat secondary antibody (1:500, Invitrogen, MA, USA) were used for immunofluorescence. Counter staining was done with DAPI. Fluorescence signal was observed with a Fluorescent microscope (Olympus, Tokyo, Japan) or confocal microscope (Zeiss, Oberkochen, Germany). For immunohistochemical experiments, anti-mouse, anti-rabbit, and anti-rat HRP-conjugated antibodies (1:500, Santacruz, CA, USA) were utilized. Secondary antibodies were incubated for $2 \mathrm{hr}$ at room temperature. DAB chromogen was used for color reaction (Dako Glostrup, CA, USA) and counter staining was done with hematoxylin. Image signal was observed with an optical microscope (Olympus, Tokyo, Japan).

\section{5-bromodeoxy-uridine (BrdU) labeling}

For Label-retaining cell experiments, 10-day old mice were injected with 5-bromodeoxy-uridine (BrdU, $50 \mu \mathrm{g} / \mathrm{g}$ ) intraperitoneally (i.p) every $12 \mathrm{hr}$ for $48 \mathrm{hr}$ [26]. After a 60-day chase period, the dorsal skin was isolated. For proliferation analysis, $\mathrm{BrdU}(150 \mu \mathrm{g} / \mathrm{g})$ was i.p injected to mice $1 \mathrm{hr}$ prior to sacrifice at the indicated days.

\section{qRT-PCR}

Total RNA was extracted from either dorsal skin or epidermis using Trizol reagent (Invitrogen, Carlsbad, CA, USA) following the manufacturer's instructions. The single-stranded cDNAs were synthesized using 
the Prime Script $1^{\text {st }}$ strand cDNA Synthesis kit (Takara, Tokyo, Japan) and used for real time PCR using a CFX96 Touch $^{\mathrm{TM}}$ Real-Time PCR Detection System (Bio-Rad Laboratories, Hercules, CA, USA). Primer sequences for each gene are listed in table S1.

\section{FACS analysis}

Mouse epidermis were isolated from back skin of $1^{\text {st }}$ and $2^{\text {nd }}$ telogen mice as described previously [27]. Cells were labeled with anti-integrin a6 antibody conjugated to phycoerythrin (BD Bioscience, $\mathrm{NJ}$, USA)and anti-CD34 conjugated to FITC (eBiosciences, Waltham, MA, USA) for 30 min on ice. Cells were then analyzed using BD FACS Cantoll sorter and FACSDiva ${ }^{\text {TM }}$ software analysis system.

\section{Statistical analysis}

$P$ values were determined using Student's t-tests. Statistical significance was set at $p<0.05$.

\section{Results}

\section{Alteration in hair cycling of the hairpoor mouse}

The hairpoor mouse exhibited increased proliferation and differentiation of epidermal cells [24] and premature catagen followed by the early telogen at the first hair cycle [25], indicating aberrant HF development. Because the hairpoor mice display abnormal hair loss with aging similarly to MUHH patients, we hypothesized that HFSC may be affected in this disorder leading to abnormal HF cycle, resulting in acceleration of hair loss.

To investigate this hypothesis, we first observed and compared the hair cycle between the hairpoor and the littermate wildtype mice, including the second telogen stage (Fig. S1 and Fig. 1a). H\&E staining showed that a much shortened anagen stage following the premature catagen and earlier onset of the 1st telogen in the hairpoor mouse compared to that of the wild type mouse. At P19-20, the HF of $+/ H r^{H p}$ was at the $1^{\text {st }}$ telogen stage, while HF of the wild type mouse was still at the $1^{\text {st }}$ catagen, showing the earlier onset of telogen in the hairpoor mouse. Due to the shortened cycle, the onset of the $2^{\text {nd }}$ telogen was much earlier and furthermore its duration was remarkably shorter in the hairpoor mouse compared to the wild type mouse. The $2^{\text {nd }}$ telogen started at P35 and lasted for approximately one day (P35-P36) in the hairpoor HF, while it started at P42 and lasted for more than 2 weeks in the wild type HF (Fig 1a).

Among the telogen phased HFs of the hairpoor mouse, the clear morphological abnormality was not observed compared to the wild type HF as shown (Fig. 1b). However, HFs' stages in the hairpoor mouse were much differed from that of the wild type mouse. At the $1^{\text {st }}$ telogen, the proportion of telogen phased HF was $91.5 \%$ in the wild type mouse whereas it was only $58.3 \%$ in the hairpoor mouse. In the case of the $2^{\text {nd }}$ telogen, all the wild type HF was at telogen phase, but only about $30 \%$ of the hairpoor HF were at telogen phase and $70 \%$ were already into the anagen phase (Fig. 1c). Following the short $2^{\text {nd }}$ telogen, the 
HFs were a mixture of mostly anagen, a few catagen, and telogen phased HFs in the hairpoor mouse (data not shown).

Interestingly, the number of HF did not differ significantly between the wild type and the hairpoor mouse until P21. However, at P28, the HF number in $+/ H r^{H p}$ was found to be drastically lower than that of the wild type mouse and the difference was maintained throughout the observed period (Fig. 1d), suggesting that the HFs' regeneration capability seemed to have been affected by the first telogen and those defects persisted in the hairpoor mouse. These results clearly indicated that the transition of the stages of HF cycle was abnormal in the hairpoor mouse and raised a possibility of the impairment of HFSC that controls the HF cycle.

\section{Decrease in the number of CD34 ${ }^{+}$HFSC $n$ hairpoor mice}

In order to dissect the abnormal hair cycle in the hairpoor mouse, we investigated whether there was any changes in HFSCs. First, the number of bulges expressing K15, a bulge marker, and CD34, a HFSC marker, [28], was determined at telogen stages using immunohistochemistry. At both telogen stages, all K15 ${ }^{+}$ bulges were shown to have similar intensities for both the wild type and the hairpoor mice (Fig. 2a). In contrast to K15, CD34 expression displayed a different pattern between two lines. Almost all the K15 ${ }^{+}$ bulges were $\mathrm{CD} 34^{+}$at the first telogen (7.25/7.5) and the second telogen (6.8/6.8) stages of the wild type HFs. In contrast, on average, only $45 \%(3.5 / 7.75)$ and $70 \%(3.8 / 5.4)$ of the $\mathrm{K} 15^{+}$bulges showed CD $34^{+}$ expression at the first and second telogen of the hairpoor HFs, respectively. When the number of CD34 ${ }^{+}$ bulges were determined, the bulge showing detectable expression level of CD34 was considered as CD34 $4^{+}$bulge. (Fig. 2b). In addition to reduced number of $\mathrm{CD} 34^{+}$bulges, there was much weaker expression in the CD34 $4^{+}$bulges, of the hairpoor HFs compared to those of the wild type HFs (Fig. 2a, b). Interestingly, the proportion of $\mathrm{CD} 34^{+} / \mathrm{K} 15^{+}$bulges was higher at $2^{\text {nd }}$ telogen than at the $1^{\text {st }}$ telogen of the hairpoor HFs, although the expression level was much lower than that of the wild type HF,

To assess the extent of the reduced expression of CD34 in the hairpoor HFs, Cd34 mRNA expression level was determined using qRT-PCR. Relative expression level of Cd34 mRNA was significantly reduced in the hairpoor epidermis compared to that of the wild type (Fig. 2c), with less than $20 \%$ of that of the wild type mouse for both telogen stages.

We next determined the proportion of HFSCs $\left(\operatorname{ITGA}^{+} / \mathrm{CD} 34^{+}\right)$in the bulge at both telogen stages using FACS analysis (Fig. 2d, e). In wild type mice, ITGA6 ${ }^{+} / \mathrm{CD} 34^{+}$cells composed of $5.2 \%$ and $9.6 \%$ of the bulge cells at the $1^{\text {st }}$ and $2^{\text {nd }}$ telogen stages, respectively. In contrast, the bulges in hairpoor mice contained only $0.2 \%$ and $1.8 \% \mathrm{ITGA} 6^{+} / \mathrm{CD} 34^{+}$cells of the bulge cells at the $1^{\text {st }}$ and the $2^{\text {nd }}$ telogen stages, respectively. This result clearly indicates that the number of HFSCs expressing CD34 was significantly reduced in the hairpoor mouse. Taken together, the hairpoor mouse contained much fewer HFSCs present in the HFs compared to the wild type, suggesting aberrance in the maintenance of HFSC in the hairpoor mouse. 


\section{Loss of HFSC quiescence and increased cell proliferation in the hairpoor HF}

In order to investigate whether the quiescence of HFSC was disturbed in the hairpoor mouse, we first determined the number of label-retaining cells (LRC) in HF using 5-bromodeoxy-uridine (BrdU) incorporation method at 60 days post an injection of BrdU at P10. There was less than one BrdU ${ }^{+} \mathrm{LRC}$ detected in the bulge of hairpoor HF while the wild type HF contained three BrdU ${ }^{+}$LRCs on average (Fig. $3 a, b)$. We then determined cell proliferation in HFs to delineate whether HFSCs lost quiescence. Cell proliferation was assessed by measurement of the BrdU incorporation in cells $1 \mathrm{hr}$ after BrdU injection. The experiments were carried out at anagen I-II period for both wild type and hairpoor mice and revealed that the hairpoor mouse exhibited $15 \mathrm{BrdU}^{+}$cells/HF on average while the wild type mouse showed 0.48 $\mathrm{BrdU}^{+}$cells/HF (Fig. 3c, d), showing excessive cell proliferation in the hairpoor HFs.

To further characterize HFSCs in the hairpoor mouse, the histological study was conducted on skin sections prepared after synchronization of HF cycling via depilation at the $2^{\text {nd }}$ telogen phase. As shown in the Fig. 4a, HF cycle of the hairpoor mouse was much shorter than that of the wild type mouse. At 14 days post depilation, while the wild type HFs were still in the full anagen phase, the hairpoor HFs were into the catagen phase (Fig. 4a). This deviation in the hair cycle of the hairpoor mouse was more prominent in subsequent days. Specifically, at 21 days post depilation, the hairpoor HFs were in the anagen phase again whereas the wild type HFs were at the late catagen/early telogen phase, indicating that the telogen stage of the hairpoor HFs passed between 14 and 21 days post depilation. In contrast, the wild type HFs began the telogen stage at 21 days remain in the same stage at 28 days post depilation while the hairpoor HFs were in anagen stage again..

Cell proliferation in HFs was examined at 7 days after depilation when both the wild type and the hairpoor HFs were in anagen phase. Immunohistochemical detection of Ki67 indicated higher proliferation of the cells in the bulge and bulb of the hairpoor HFs compared to those of the wild type HFs (Fig. 4b). In addition, expression of Lymphoid enhancer-binding factor 1 (LEF1), transcription activator in the presence of $B$-catenin, was expressed at both the bulges of $1^{\text {st }}$ and $2^{\text {nd }}$ telogen staged HFs of the hairpoor mouse (Fig. 4c). Taken together, the HFSCs in the hairpoor mouse were not able to maintain the quiescence, resulting in proliferation of the cells in HF.

\section{Expression of HFSC markers in the hairpoor mice}

To dissect the status of the HFSC of the hairpoor mice, expression of various HFSC markers were observed including LIM homeobox 2 (LHX2), SRY-box 9 (SOX9), Nuclear factor of activated T cell 1 (NFATc1), and Transcription factor 4 (TCF4) [29-33] by immunohistochemstry. LHX2, expressing in the bulge, plays an essential role in $\mathrm{HF}$ morphogenesis as well as in maintenance of HFSC quiescence. SOX9 is known to be associated with Shh signaling and expressed in the bulge and outer root sheath. As shown in Fig $5 a$ and $5 b$, expressions of LHX2 and SOX9 were not significantly different in the $1^{\text {st }}$ telogen and $2^{\text {nd }}$ telogen phases of both the wild type and the hairpoor mouse HFs. Expression of other bulge markers, NFATC1 and TCF4, were also detected in the junctional zone and interfollicular epidermis, and the bulges, 
respectively. TCF4, shown to suppress HFSC quiescence and thus inhibit transition of HFs from telogen to anagen, was also expressed in the bulge. As with LHX2 and SOX9, no noticeable difference was observed in NFATc1 and TCF4 expressions between the hairpoor and the wild type HFs. Thus, expression of all the HFSC markers tested in this study with exception of CD34 did not show differences between the hairpoor and the wild type.

\section{Disruption of the HFSC quiescence signaling in the hairpoor mouse}

Next, we investigated whether the HFSC niche signaling was involved in the abnormal hair cycling of the hairpoor mouse. We observed the expression of K6, a HFSCs niche marker, by IHC and found that all the $\mathrm{K}^{1} 5^{+}$bulges showed $\mathrm{K}^{+}$in the hairpoor HFs as the same as in the wild type (Fig. S2). We also observed expression of $B m p 6$ and $F g f 18$ in the epidermis of mice, since $\mathrm{K}^{+}{ }^{+}$was shown to regulate the quiescence of HFSC through expression of Bmp6 and Fgf18 [12]. Expression of Bmp6 and Fgf18 displayed a different pattern from each other. Bmp6 expression was significantly decreased only in the $2^{\text {nd }}$ telogen phase in the hairpoor HFs compared to that of the wild type HFs. In contrast to Bmp6, Fgf18 expression was significantly decreased in both $1^{\text {st }}$ and $2^{\text {nd }}$ telogen phases in the hairpoor HFs compared to the wild type ones (Fig. 6a, b and c). These findings demonstrate that reduced expression of Fgf18 and Bmp6 concurred with disturbance of the HFSC quiescence in the hairpoor mice, suggesting that the lack of expression of the quiescence maintaining regulators may cause disarrayed HF cycling. Next, we dissected whether the $\mathrm{HF}$ in the telogen phase of the hairpoor mouse was in the competent telogen state or the telogen-anagen transition state [4] by determining the expression of the Wnt signaling molecules in the skin. Expression of Sfrp1, a Wnt inhibitor, was reduced in the $2^{\text {nd }}$ telogen of the hairpoor HFs to $40 \%$ of that of the wild type (Fig6 d) and expression of Wnt7b was increased in both $1^{\text {st }}$ and $2^{\text {nd }}$ telogen in the hairpoor compared to the wild type (Fig6 e). In addition, expression of Axin2 and Wnt10b mRNA was remarkably increased in the $2^{\text {nd }}$ telogen of the hairpoor mouse compared to the wild type mouse (Fig. $6 f$, g). These observations suggest that overexpression of HR in the hairpoor mouse led to the reduction of HFSC quiescence signaling molecules in HFSC niche, and concomitantly activated the Wnt signaling pathway, resulting in transition of the hairpoor HFs from telogen to telogen-anagen transition state.

\section{Discussion}

$\mathrm{HR}$ is expressed in HF and regulates downstream gene expression as a transcriptional co-factor [17, 34]. In skin, HR is known to regulate the HF cycle by regulating Wise expression by being involved in Wnt signaling [34]. However, association of HR with HFSC has not been explored. Here we report that HR overexpression resulted in completely disrupted HF cycle which may be caused by abnormal expression of molecules involved in HFSC regulation in HF niche using the hairpoor mouse.

The hairpoor mouse $\left(+/ H r^{H p}\right)$ is a human MUHH disease model in which this heterozygote displays scarce hair at young age then gradual progression to baldness with aging as $\mathrm{MUHH}$ patients manifest $[23,24]$. Previously, we reported that the length of HF was shorter, and a premature catagen stage followed by earlier onset of telogen stage in the first cycle of the hairpoor mouse compared to that of the 
wild type mouse [25]. The current study explored this abnormal hair cycle further and found that the abnormality of the early HF cycle is accentuated in the 2nd cycle. The 2nd cycle of the hairpoor HF displayed a much shorter anagen stage and a lack of stable telogen stage which was composed of a mixed population of anagen and telogen phased HFs whereas the wild type HFs showed the stable and long 2nd telogen stage with all HFs at telogen phase (Fig. 1, Fig. S1). In addition, the numbers of HFs were found to be reduced in the 4 week old mice which persisted till at least P56 of the hairpoor mice. This phenomenon continued and the decrease in HF number became greater in mice older than 1 year (data not shown). These results indicate that overexpression of $\mathrm{Hr}$ disturbed not only the first cycle but also the following cycles, suggesting Hrinvolvement in the status of HFSC.

Expression of stem cell specific markers provides valuable information in determination of the status of stem cells. Of the HFSC associated markers investigated in this study, all the markers including NFATc1, SOX9, LHX2, and TCF4 with exception of CD34 expressed in all the $\mathrm{K}^{2} 5^{+}$bulges of the hairpoor HFs. Expression of these HFSC markers has been shown to determine the quiescent state or in transition from telogen to anagen in $\operatorname{HF}[12,28,30-32,35]$. Loss of SOX9 was reported to cause failure in maintaining the HFSC quiescence in conjunction with reduced CD34 expression [36]. Interestingly, the HFSC of the hairpoor mouse exhibited reduced CD34 expression while SOX9 expression was not affected compared to the wild type HFSC. In addition, the expression of LHX2 which is expressed in both embryonic hair placodes and postnatal HFSC [30], has no significant difference in the hairpoor mouse. Surprisingly, only CD34 showed the dramatically reduced expression at the first telogen HFs in the hairpoor mouse. CD34, the most widely used HFSC marker, is known to express in the bulges including the 1st telogen stage [37, $38]$ and its expression has been shown to be essential in HFSCs' integrity [39, 40]. Thus, reduction in CD34 expression in the bulges of the 1st telogen staged HFs strongly suggested the loss of the quiescence of the HFSC. The loss of HFSC quiescence was further supported by the accelerated cell proliferation in the hairpoor HFs and recapitulation of the same results as shown in the disarrayed hair cycle in the depilation experiment.

Due to the lack of differences in HFSC marker expression other than CD34, we further investigated expression of the niche markers. While the expression of a HFSC niche marker, K6, was not changed, expression of Bmp6 and Fgf18, which are known to maintain telogen state [41, 42], was found to have a dramatic reduction in the hairpoor mouse. Interestingly, at the 1 st telogen, Fgf18 had a distinctly reduced expression level whereas Bmp6 did not. Recently, Foxp1 was shown to maintain HFSC quiescence through Fgf18 without altering Bmp6 expression [43]. Our results clearly add the evidence that $B m p 6$ and Fgf18 expressed in the $\mathrm{K}^{+}{ }^{+}$inner bulge play different roles in maintaining HFSC quiescence. The relationship between $\mathrm{Hr}$ and Foxp 1 remains to be explored.

During the telogen, Sfrp1, a Wnt inhibitor, was shown to express in both inner and outer bulges and be in balance with Wnt7b expression in the niche cells with Axin2 expression in maintaining the HFSC quiescence [44]. In the hairpoor mouse, Sfrp 1 expression was markedly decreased in the 2nd telogen. We have shown that HR expression suppressed Sfrp1 mRNA expression [45]. Thus, presumably HR overexpression partly contributes to loss of HFSC quiescence via down regulation of Sfrp 1 expression in 
the hairpoor mouse. Activation of the Wnt signaling pathway during the 2nd telogen was demonstrated by increased expression of Axin2, Wnt7b and Wnt10b, which explains the findings that a large portion of HFs were in the telogen-anagen transition state in hairpoor mice. In addition, we previously documented that another Wnt inhibitor, Dickkopf-related protein 1 (Dkk1) was overexpressed in hairpoor mouse [25]. Dkk1 was shown to express at anagen and catagen HFs and known to induce HF from anagen to catagen [46]. These findings together strongly suggest that the acceleration of HF cycling was led by the aberrant Wnt signaling in hairpoor mouse. This may be related to development of alopecia with aging in the hairpoor mouse and further study is required to better elucidate this disorder.

\section{Conclusions}

In conclusion, in the HR overexpressing hairpoor mice, the HFSC quiescence is not maintained due to decreased HFSC niche signals including Fgf18, Bmp6, and Sfrp1. This leads to the telogen to anagen transition of HFs at the telogen phase and HF proliferation is increased through activation of the Wnt signaling in the hairpoor mouse. Consequently, this causes abnormalities of HF cycling, which eventually leads to alopecia. Our results indicate that HR plays an important role in HFSC quiescence by regulating the signaling networks in HF. Our study elucidates the molecular genetic etiology of MUHH disease and may provide therapeutic directions.

\section{Abbreviations}

HFSC: Hair follicle stem cell; HF: Hair follicle; Hr: Hairless; MUHH: Marie-unna hypotrichosis; LRC: label retaining cell; BMP: Bone morphogenetic protein; K6: Keratin 6; BrdU: 5-bromodeoxy-uridine; LEF1: Lymphoid enhancer-binding factor1; LHX2: LIM homeobox 2; SOX9: SRY-box 9; NFATc1: Nuclear factor of activated T cell 1; TCF4: Transcription factor 4, Dkk1: Dickkopf-related protein 1.

\section{Declarations}

\section{Acknowledgment}

Not applicable

\section{Author Contributions}

K-C and SK-Y planned and designed experiments, performed experiments, data analysis and wrote the manuscript. SH-P analyzed FACS data and provision of study material. SY-P analyzed qRT-PCR and generated hairpoor mice.

\section{Funding}

This study was supported by the Ministry of Health and Welfare, Republic of Korea (grant number: $\mathrm{HI} 17 \mathrm{C0616}$ ) and the National Research Foundation of Korea (NRF) funded by the Ministry of Education, 
Science and Technology (NRF-2021R1F1A1060935).

Availability of data and materials

The dataset used and/or analyzed during the current study are available from the corresponding author upon reasonable request.

Ethics approval and consent to participate

All experiments were performed according to standard protocols, in compliance with the Guidelines and Policies for Rodent Experiments provided by the IACUC (Institutional Animal Care and Use Committee) in the School of Medicine, The Catholic University of Korea.

Consent for publication

Not applicable

Competing interests

The authors declare that they have no competing interests.

Author details

${ }^{1}$ Department of Biomedicine \& Health Sciences, the Catholic University of Korea, Seoul, Republic of Korea.

${ }^{2}$ Department of Medical Life Sciences, the Catholic University of Korea, Seoul, Korea.

\section{References}

1. Greco V, Chen T, Rendl M, Schober M, Pasolli HA, Stokes N, Dela Cruz-Racelis J, Fuchs E: A two-step mechanism for stem cell activation during hair regeneration. Cell Stem Cell 2009, 4:155-169.

2. Nowak JA, Polak L, Pasolli HA, Fuchs E: Hair follicle stem cells are specified and function in early skin morphogenesis. Cell Stem Cell 2008, 3:33-43.

3. Muller-Rover S, Handjiski B, van der Veen C, Eichmuller S, Foitzik K, McKay IA, Stenn KS, Paus R: A comprehensive guide for the accurate classification of murine hair follicles in distinct hair cycle stages. J Invest Dermatol 2001, 117:3-15.

4. Plikus MV: New Activators and Inhibitors in the Hair Cycle Clock: Targeting Stem Cells' State of Competence. Journal of Investigative Dermatology 2012, 132:1321-1324.

5. Choi YS, Zhang Y, Xu M, Yang Y, Ito M, Peng T, Cui Z, Nagy A, Hadjantonakis AK, Lang RA, et al: Distinct functions for Wnt/beta-catenin in hair follicle stem cell proliferation and survival and interfollicular epidermal homeostasis. Cell Stem Cell 2013, 13:720-733.

6. Lien W-H, Fuchs E: Wnt some lose some: transcriptional governance of stem cells by Wnt/ $\beta$-catenin signaling. Genes \& Development 2014, 28:1517-1532. 
7. Kandyba E, Kobielak K: Wnt7b Is an Important Intrinsic Regulator of Hair Follicle Stem Cell Homeostasis and Hair Follicle Cycling. STEM CELLS 2014, 32:886-901.

8. Shen Q, Yu W, Fang Y, Yao M, Yang P: Beta-catenin can induce hair follicle stem cell differentiation into transit-amplifying cells through c-myc activation. Tissue and Cell 2017, 49:28-34.

9. Huelsken J, Vogel R, Erdmann B, Cotsarelis G, Birchmeier W: $\boldsymbol{\beta}$-Catenin Controls Hair Follicle Morphogenesis and Stem Cell Differentiation in the Skin. Cell 2001, 105:533-545.

10. Hsu Y-C, Li L, Fuchs E: Emerging interactions between skin stem cells and their niches. Nat Med 2014, 20:847-856.

11. Kobielak K, Stokes N, de la Cruz J, Polak L, Fuchs E: Loss of a quiescent niche but not follicle stem cells in the absence of bone morphogenetic protein signaling. Proceedings of the National Academy of Sciences 2007, 104:10063-10068.

12. Hsu Y-C, Pasolli HA, Fuchs E: Dynamics between Stem Cells, Niche, and Progeny in the Hair Follicle. Cell 2011, 144:92-105.

13. Kimura-Ueki M, Oda Y, Oki J, Komi-Kuramochi A, Honda E, Asada M, Suzuki M, Imamura T: Hair Cycle Resting Phase Is Regulated by Cyclic Epithelial FGF18 Signaling. Journal of Investigative Dermatology 2012, 132:1338-1345.

14. Plikus MV, Mayer JA, de la Cruz D, Baker RE, Maini PK, Maxson R, Chuong C-M: Cyclic dermal BMP signalling regulates stem cell activation during hair regeneration. Nature 2008, 451:340-344.

15. Moraitis AN, Giguere V, Thompson CC: Novel mechanism of nuclear receptor corepressor interaction dictated by activation function 2 helix determinants. Mol Cell Biol 2002, 22:6831-6841.

16. Potter GB, Beaudoin GMJ, DeRenzo CL, Zarach JM, Chen SH, Thompson CC: The hairless gene mutated in congenital hair loss disorders encodes a novel nuclear receptor corepressor. Genes \& Development 2001, 15:2687-2701.

17. Hsieh JC, Sisk JM, Jurutka PW, Haussler CA, Slater SA, Haussler MR, Thompson CC: Physical and functional interaction between the vitamin $D$ receptor and hairless corepressor, two proteins required for hair cycling. J Biol Chem 2003, 278:38665-38674.

18. Panteleyev AA, Paus R, Christiano AM: Patterns of Hairless (hr) Gene Expression in Mouse Hair Follicle Morphogenesis and Cycling. The American Journal of Pathology 2000, 157:1071-1079.

19. Thompson CC, Sisk JM, Beaudoin GM, 3rd: Hairless and Wnt signaling: allies in epithelial stem cell differentiation. Cell Cycle 2006, 5:1913-1917.

20. Ahmad W, Zlotogorski A, Panteleyev AA, Lam H, Ahmad M, Faiyaz ul Haque M, Abdallah HM, Dragan L, Christiano AM: Genomic organization of the human hairless gene (HR) and identification of a mutation underlying congenital atrichia in an Arab Palestinian family. Genomics 1999, 56:141-148.

21. Nam Y, Kim JK, Cha DS, Cho JW, Cho KH, Yoon S, Yoon JB, Oh YS, Suh JG, Han SS, et al: A novel missense mutation in the mouse hairless gene causes irreversible hair loss: genetic and molecular analyses of Hr m1Enu. Genomics 2006, 87:520-526. 
22. Wen Y, Liu Y, Xu Y, Zhao Y, Hua R, Wang K, Sun M, Li Y, Yang S, Zhang XJ, et al: Loss-of-function mutations of an inhibitory upstream ORF in the human hairless transcript cause Marie Unna hereditary hypotrichosis. Nat Genet 2009, 41:228-233.

23. Baek IC, Kim JK, Cho KH, Cha DS, Cho JW, Park JK, Song CW, Yoon SK: A novel mutation in Hr causes abnormal hair follicle morphogenesis in hairpoor mouse, an animal model for Marie Unna Hereditary Hypotrichosis. Mamm Genome 2009, 20:350-358.

24. Kim JK, Kim E, Baek IC, Kim BK, Cho AR, Kim TY, Song CW, Seong JK, Yoon JB, Stenn KS, et al: Overexpression of $\mathrm{Hr}$ links excessive induction of Wnt signaling to Marie Unna hereditary hypotrichosis. Hum Mol Genet 2010, 19:445-453.

25. Kim BK, Lee HY, Kim I, Choi K, Park J, Yoon SK: Increased expression of Dkk1 by HR is associated with alteration of hair cycle in hairpoor mice. J Dermatol Sci 2014, 74:81-87.

26. Braun KM, Watt FM: Epidermal Label-Retaining Cells: Background and Recent Applications. Journal of Investigative Dermatology Symposium Proceedings 2004, 9:196-201.

27. Jensen KB, Driskell RR, Watt FM: Assaying proliferation and differentiation capacity of stem cells using disaggregated adult mouse epidermis. Nat Protoc 2010, 5:898-911.

28. Trempus CS, Morris RJ, Bortner CD, Cotsarelis G, Faircloth RS, Reece JM, Tennant RW: Enrichment for living murine keratinocytes from the hair follicle bulge with the cell surface marker CD34. J Invest Dermato/2003, 120:501-511.

29. Plikus MV, Gay DL, Treffeisen E, Wang A, Supapannachart RJ, Cotsarelis G: Epithelial stem cells and implications for wound repair. Seminars in Cell \& Developmental Biology 2012, 23:946-953.

30. Rhee H, Polak L, Fuchs E: Lhx2 Maintains Stem Cell Character in Hair Follicles. Science (New York, NY) 2006, 312:1946-1949.

31. Horsley V, Aliprantis AO, Polak L, Glimcher LH, Fuchs E: NFATc1 Balances Quiescence and Proliferation of Skin Stem Cells. Cell 2008, 132:299-310.

32. Vidal VPI, Chaboissier M-C, Lützkendorf S, Cotsarelis G, Mill P, Hui C-C, Ortonne N, Ortonne J-P, Schedl A: Sox9 Is Essential for Outer Root Sheath Differentiation and the Formation of the Hair Stem Cell Compartment. Current Biology 2005, 15:1340-1351.

33. Lien W-H, Polak L, Lin M, Lay K, Zheng D, Fuchs E: In vivo transcriptional governance of hair follicle stem cells by canonical Wnt regulators. Nat Cell Bio/ 2014, 16:179-190.

34. Beaudoin GM, 3rd, Sisk JM, Coulombe PA, Thompson CC: Hairless triggers reactivation of hair growth by promoting Wnt signaling. Proc Natl Acad Sci U S A 2005, 102:14653-14658.

35. Mardaryev AN, Meier N, Poterlowicz K, Sharov AA, Sharova TY, Ahmed MI, Rapisarda V, Lewis C, Fessing MY, Ruenger TM, et al: Lhx2 differentially regulates Sox9, Tcf4 and Lgr5 in hair follicle stem cells to promote epidermal regeneration after injury. Development 2011, 138:4843-4852.

36. Kadaja M, Keyes BE, Lin M, Pasolli HA, Genander M, Polak L, Stokes N, Zheng D, Fuchs E: SOX9: a stem cell transcriptional regulator of secreted niche signaling factors. Genes \& Development 2014, 28:328-341. 
37. Blanpain C, Lowry WE, Geoghegan A, Polak L, Fuchs E: Self-Renewal, Multipotency, and the Existence of Two Cell Populations within an Epithelial Stem Cell Niche. Cell 2004, 118:635-648.

38. Trempus CS, Morris RJ, Ehinger M, Elmore A, Bortner CD, Ito M, Cotsarelis G, Nijhof JGW, Peckham J, Flagler N, et al: CD34 Expression by Hair Follicle Stem Cells Is Required for Skin Tumor Development in Mice. Cancer Research 2007, 67:4173.

39. Yang L, Wang L, Yang X: Disruption of Smad4 in Mouse Epidermis Leads to Depletion of Follicle Stem Cells. Molecular Biology of the Cell 2008, 20:882-890.

40. Bhattacharya S, Wheeler H, Leid M, Ganguli-Indra G, Indra AK: Transcription Factor CTIP2 Maintains Hair Follicle Stem Cell Pool and Contributes to Altered Expression of LHX2 and NFATC1. Journal of Investigative Dermatology 2015, 135:2593-2602.

41. Osada S-I, Minematsu N, Oda F, Akimoto K, Kawana S, Ohno S: Atypical Protein Kinase C Isoform, aPKC $\lambda$, Is Essential for Maintaining Hair Follicle Stem Cell Quiescence. Journal of Investigative Dermatology 2015, 135:2584-2592.

42. Peters F, Vorhagen S, Brodesser S, Jakobshagen K, Brüning JC, Niessen CM, Krönke M: Ceramide Synthase 4 Regulates Stem Cell Homeostasis and Hair Follicle Cycling. Journal of Investigative Dermatology 2015, 135:1501-1509.

43. Leishman E, Howard JM, Garcia GE, Miao Q, Ku AT, Dekker JD, Tucker H, Nguyen H: Foxp1 maintains hair follicle stem cell quiescence through regulation of Fgf18. Development 2013, 140:3809.

44. Lim X, Tan SH, Yu KL, Lim SB, Nusse R: Axin2 marks quiescent hair follicle bulge stem cells that are maintained by autocrine Wnt/beta-catenin signaling. Proc Natl Acad Sci U S A 2016, 113:E14981505.

45. Kim B-K, Baek I-C, Lee H-Y, Kim J-K, Song H-H, Yoon SK: Gene expression profile of the skin in the 'hairpoor' (HrHp) mice by microarray analysis. BMC Genomics 2010, 11:640.

46. Kwack MH, Kim MK, Kim JC, Sung YK: Dickkopf 1 Promotes Regression of Hair Follicles. Journal of Investigative Dermatology 2012, 132:1554-1560.

\section{Figures}


a

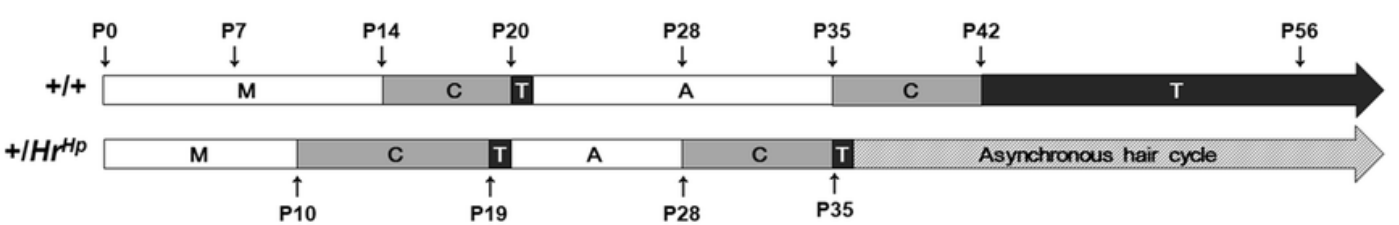

b
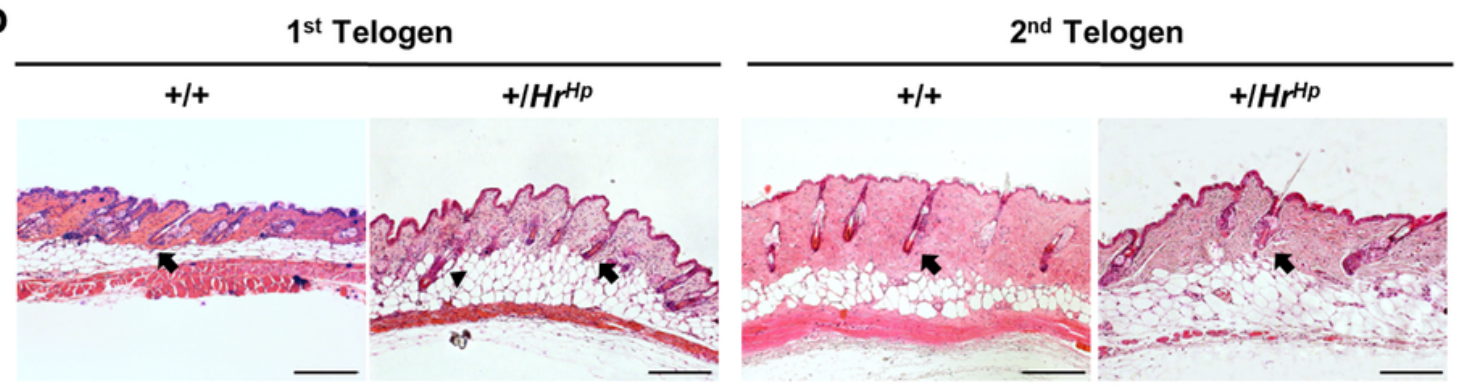

c

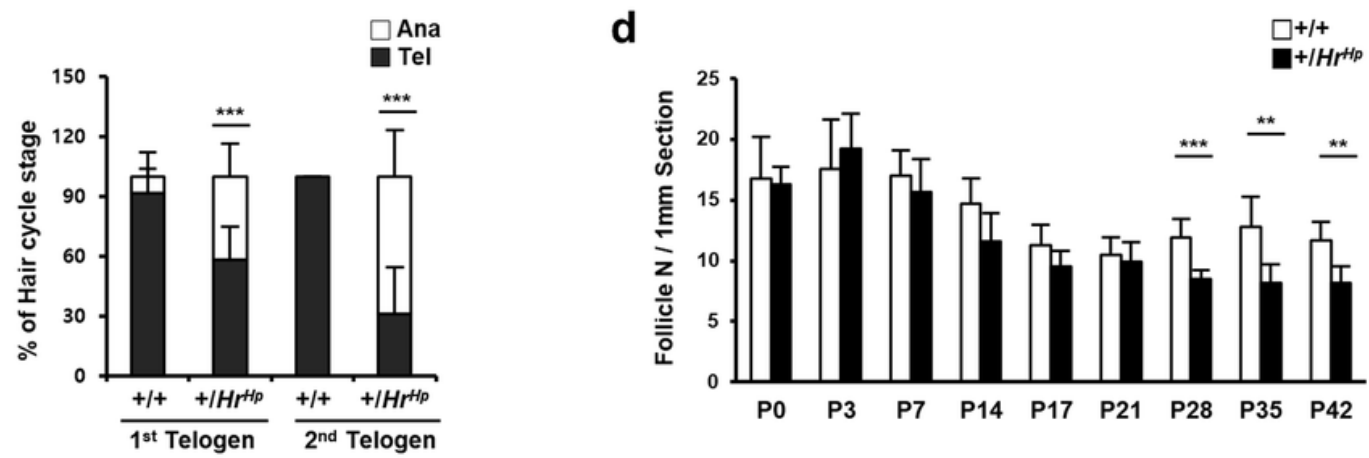

\section{Figure 1}

Histological analysis of hair cycle in hairpoor mouse. (a) Schematic presentation of the hair cycle.

Morphogenesis, M; catagen, C; telogen, T; anagen, A (b) Hematoxylin and eosin (H\&E) stain of the 1st and 2nd telogen HFs in the hairpoor mouse and the wild type mouse $(+/+)$. Arrow indicates telogen follicle and arrowhead anagen follicle. (c) Percentage of HFs of each stage of the hair cycle at the indicated telogen phase. Mouse number $n \geq 5$ per genotype. Number of HFs counted in each mouse $>20$. (d) Number of hair follicles per $1 \mathrm{~mm}$ section of back skin. Data are mean $\pm S D$. $n \geq 5$ mice per genotype/group. Scale bar $=200 \mu \mathrm{m}$. Data are mean $\pm \mathrm{SD}$. ${ }^{\star} \mathrm{P}<0.05,{ }^{\star} \star \star \mathrm{P}<0.001$. 
a

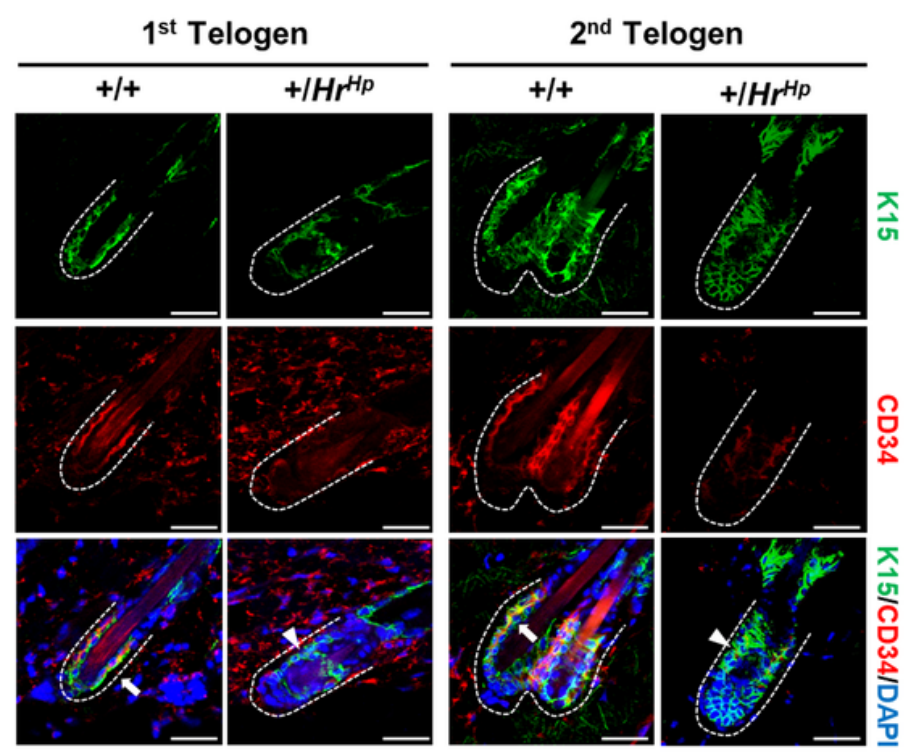

b

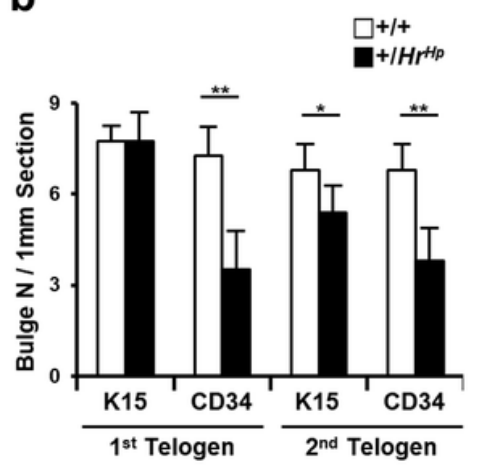

d

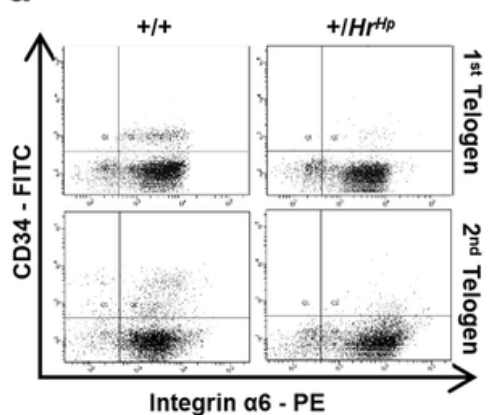

C

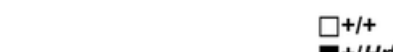

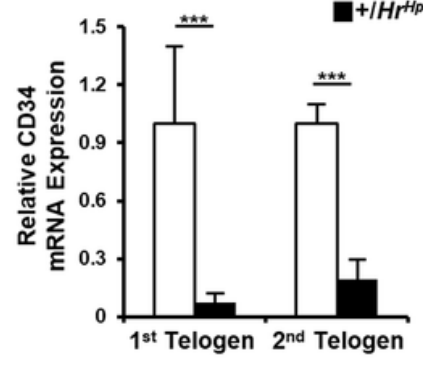

e

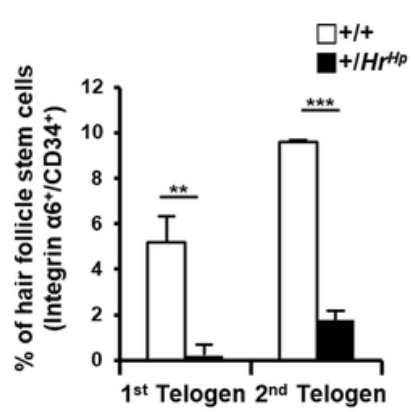

\section{Figure 2}

Decreased expression of CD34, a hair follicle stem cell (HFSC) marker, in the hairpoor mouse. (a) Immunofluorescence analyses of K15 (green) and CD34 (red) in wild type and hairpoor mice at the telogen phases. The arrow indicates K15 and CD34 doubly positive bulges and the arrowhead K15 only positive bulges. Nuclei were counterstained with DAPI. Bulge, Bu; Scale bar $=50 \mu \mathrm{m}$. (b) Number of K15 or CD34 positive HFs per $1 \mathrm{~mm}$ section of the back skin of wild type and hairpoor mouse. (Mouse number $\mathrm{n}$ $\geq 4$ per genotype). Data are mean $\pm S D$. ${ }^{*} P<0.05, * * P<0.01$. (c) qRT-PCR analysis of Cd34 in the hairpoor and the wild type mouse. (Mouse number $n \geq 3$ per genotype). Expression level of $\mathrm{Cd} 34$ was normalized against that of Gapdh and relative expression level was determined using one of the wildtype levels as reference. Data are mean $\pm S D$. ${ }^{* *} \mathrm{P}<0.001$ (d) Representative result of the flow cytometry analysis at telogen phases. (e) Proportion of the ITGA6+/CD34+ HFSCs in the epidermal cells prepared from the back skins of the hairpoor and wild type mouse. Data are mean $\pm S D$. $n=3$ mice per genotype. ${ }^{* * P} P 0.01, * \star \star P$ $<0.001$. 

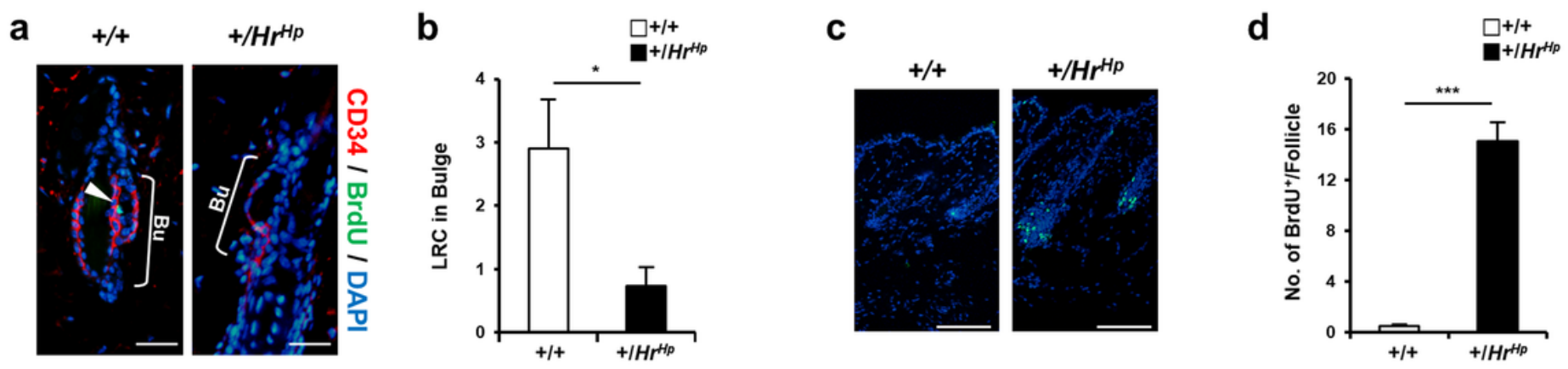

Figure 3

hairpoor mouse displays the loss of label-retaining HFSC and increased cell proliferation in HF. (a) Immunofluorescence staining of BrdU-LRC in the CD34+ bulge at P70. White arrowhead indicates LRC in bulge. Bulge, Bu; Scale bar $=25 \mu \mathrm{m}$. (b) Average number of LRC in bulge at P70. Data are mean $\pm S D$. *P $<0.05$. Mouse number $n=3$ per genotype. Observed follicle number per each mouse $n=10$ (c) Immunofluorescence staining of BrdU (1 hr chase) at anagen II HFs (the wild type mouse at P23 and the hairpoor mouse at P21). Scale bar $=100 \mu \mathrm{m}$. $(\mathrm{a}, \mathrm{c})$ Nuclei were counter stained with DAPI. (d) Quantification of BrdU positive cells in follicle. Data are mean \pm SD. ${ }^{*} P<0.01,{ }^{\star} * \star P<0.001 . n=3$ per genotype. Number of HFs counted in each mouse $\geq 10$ 


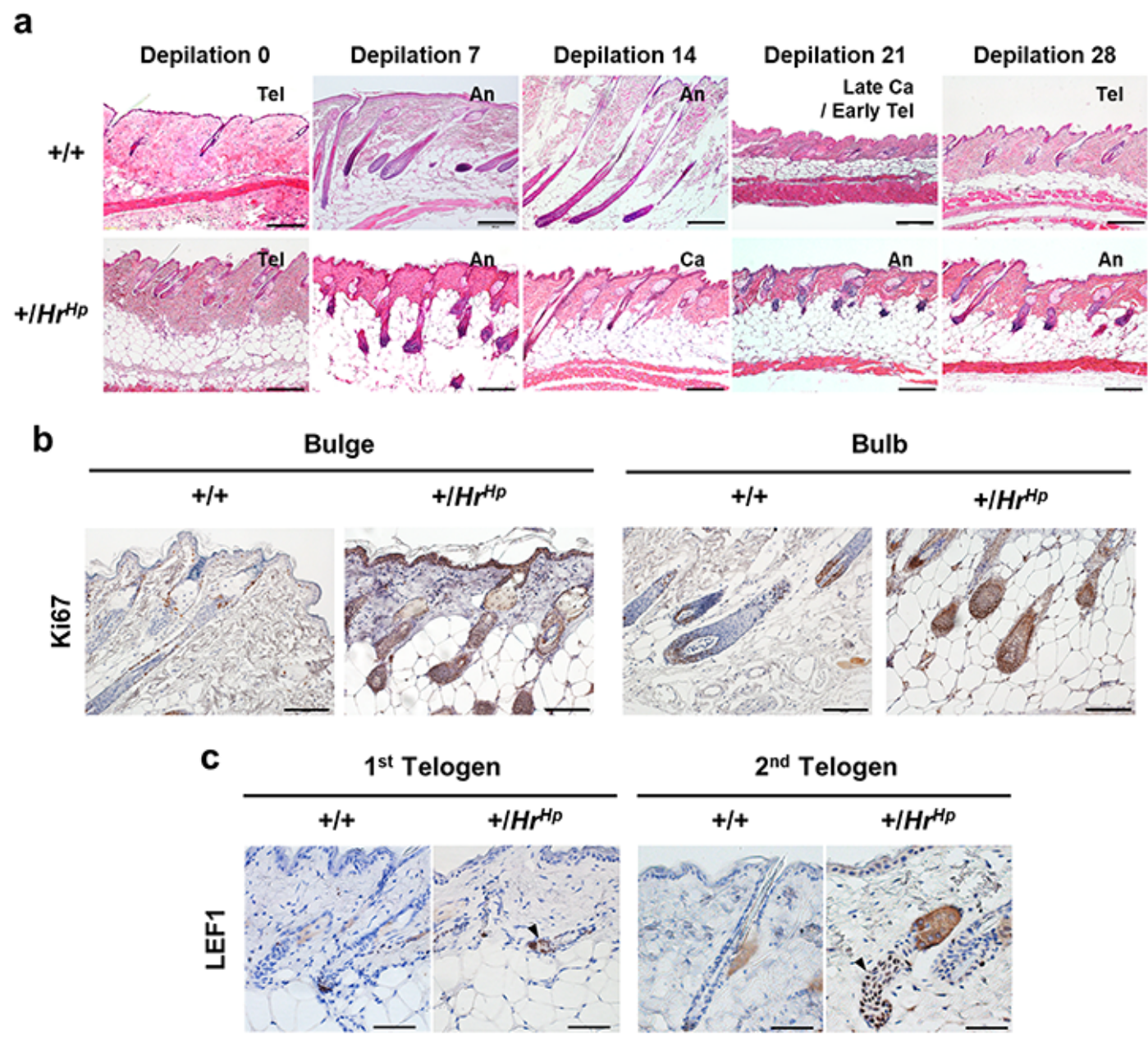

\section{Figure 4}

The hair cycle of the hairpoor mouse after depilation is impaired. (a) Histological analysis of hair cycle after depilation using H\&E staining. Telogen, Tel; anagen, An; catagen, Ca; Scale bar=200 $\mu \mathrm{m}$. (b) Immunohistochemical analysis of Ki67 in bulge and bulb using $6 \mu$ m-thick sections of the wild type and the hairpoor mouse at 7 days after depilation. Scale bar $=100 \mu \mathrm{m}$. (c) Immunohistochemical staining of LEF1 at 1st and 2nd telogen phase. Arrowhead indicates positive staining. Scale bar $=50 \mu \mathrm{m}$. 
a
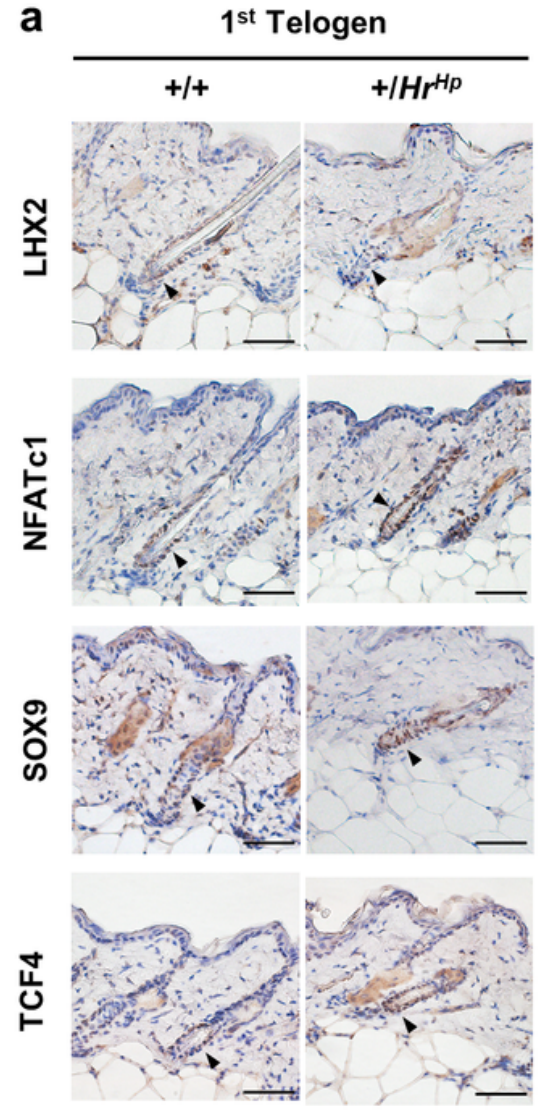

b
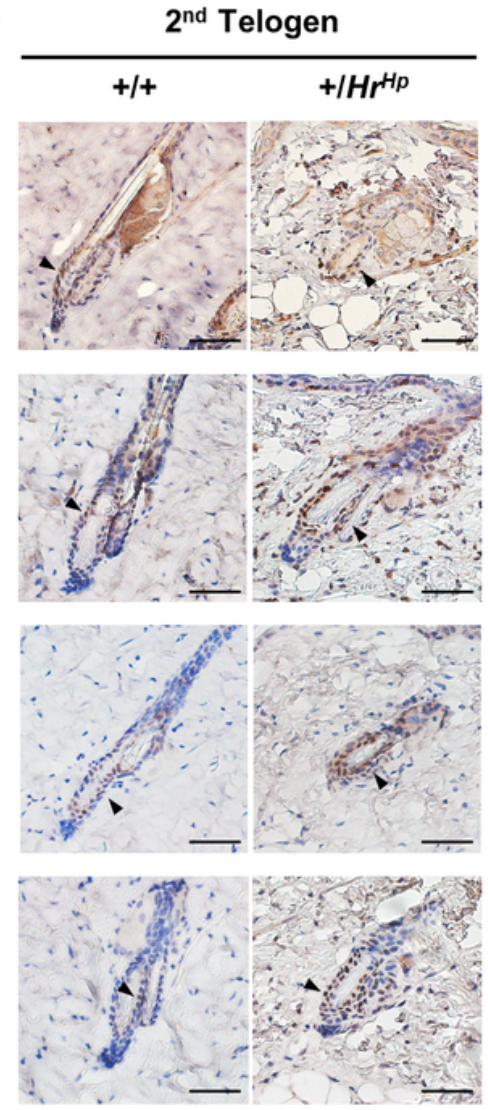

\section{Figure 5}

Expression of the HFSC quiescence markers in the hairpoor mouse. $(a, b)$ Immunohistochemical staining of the bulge HFSC markers LHX2, NFATc1, SOX9, and TCF4, at 1st (a) and 2nd (b) telogen. Arrowhead indicates expression of each marker in the bulge (dark brown). All scale bar $=50 \mu \mathrm{m}$. 


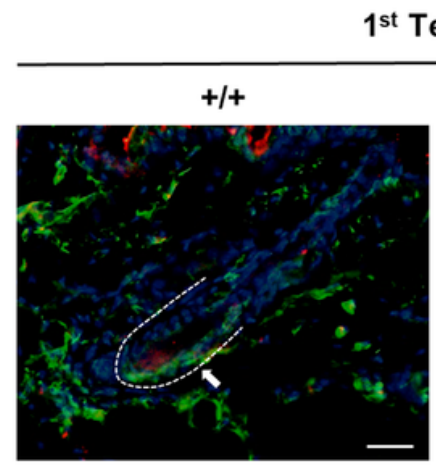

b

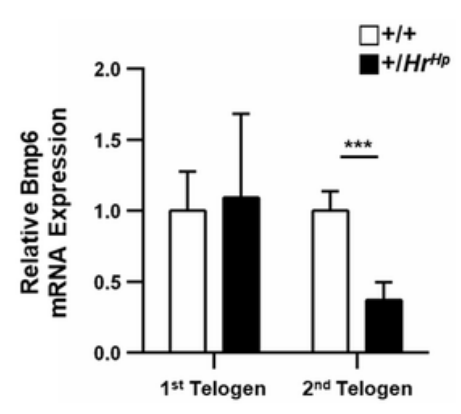

f

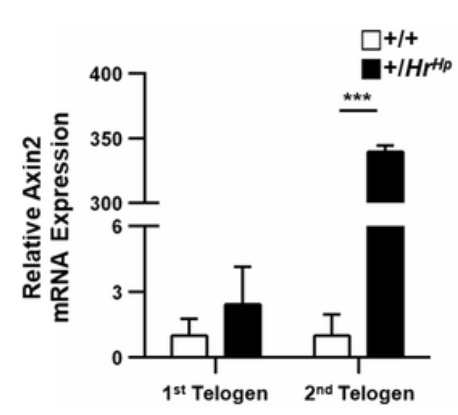

C

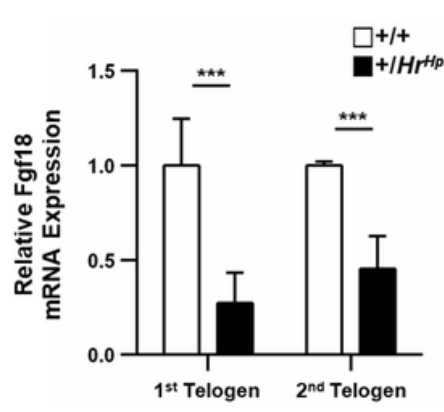

g

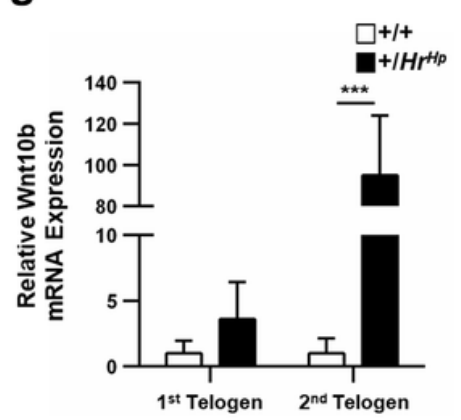

$2^{\text {nd }}$ Telogen

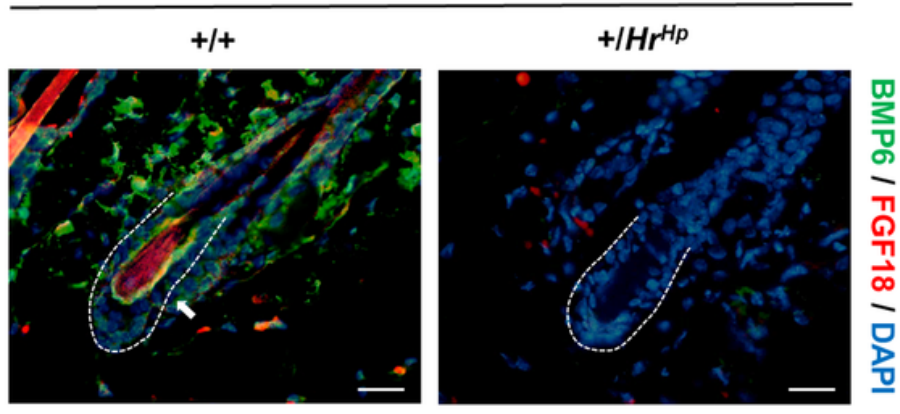

d

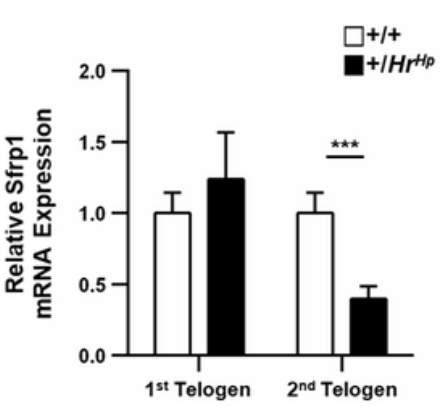

e

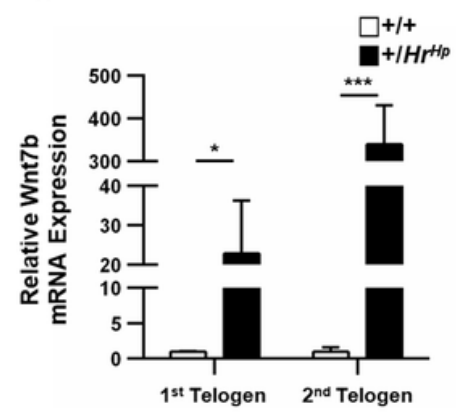

h
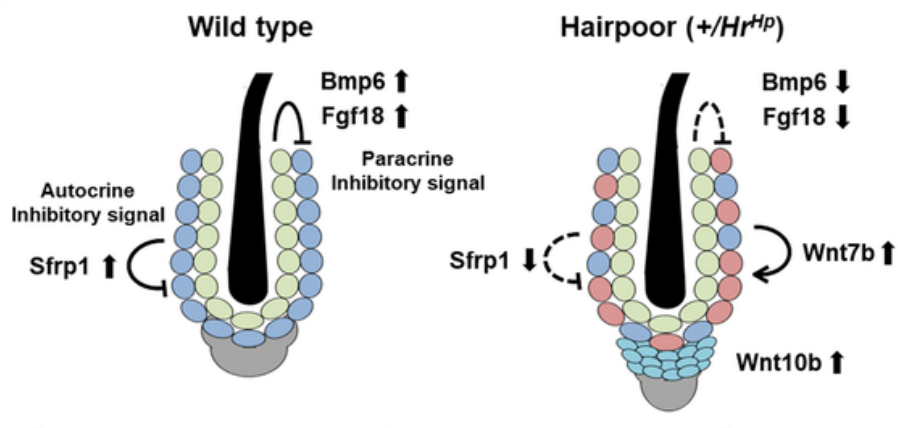

HFSC niche (K6*)

Quiescent Bulge cell (CD34 $\left.{ }^{+}\right)$

Activating Bulge cell (CD34-)

HF lineage Dermal papilla

\section{Figure 6}

Expression of Bmp6, Fgf18, and Wnt signaling molecules is disturbed in hairpoor mouse. (a) Immunohistochemical staining of BMP6 (green) and FGF18 (red) in telogen phage. The arrow indicates BMP6 and FGF18 doubly positive cells and the arrowhead BMP6 only positive cells. Nuclei were counterstained with DAPI. Bulge. Scale bar $=50 \mu \mathrm{m}$. $(\mathrm{b}, \mathrm{c})$ Expression level of Bmp6 and Fgf18 of the epidermis at the 1st and 2nd telogen phase of the hairpoor and the wild type mouse determined by qRTPCR analyses. (d,e,f,g) Expression level of the Wnt signaling molecules (Sfrp1, Axin2, Wnt7b, Wnt10b) in the telogen phased total skin of the hairpoor and wild type mouse determined by qRT-PCR analyses. $(\mathrm{h})$ Schematic of the telogen status of each mouse. mRNA expression level was normalized against that of Gapdh and relative expression level was determined using one of the wildtype levels as reference. $n \geq 3$ per genotype. Data are mean $\pm \mathrm{SD}$. ${ }^{*} \mathrm{P}<0.05$, ${ }^{* *} \mathrm{P}<0.001$ 


\section{Supplementary Files}

This is a list of supplementary files associated with this preprint. Click to download.

- Additionalfile.docx

- Supply1.tif

- Supply2.tif 